Jens Kuhle*, Christian Barro, Ulf Andreasson, Tobias Derfuss, Raija Lindberg, Åsa Sandelius, Victor Liman, Niklas Norgren, Kaj Blennow ${ }^{\mathrm{a}}$ and Henrik Zetterberg ${ }^{\mathrm{a}}$

\title{
Comparison of three analytical platforms for quantification of the neurofilament light chain in blood samples: ELISA, electrochemiluminescence immunoassay and Simoa
}

DOI 10.1515/cclm-2015-1195

Received December 2, 2015; accepted March 7, 2016; previously published online April 12, 2016

\section{Abstract}

Background: Neuronal damage is the morphological substrate of persisting neurological disability. Neurofilaments (Nf) are specific cytoskeletal proteins of neurons and their quantification has shown encouraging results as a biomarker for axonal injury.

Methods: We aimed at comparing a widely used conventional ELISA for Nf light chain (NfL) with an electrochemiluminescence-based method (ECL assay) and a newly developed single-molecule array (Simoa) method in clinically relevant cerebrospinal fluid (CSF) and serum samples.

Results: Analytical sensitivity was $0.62 \mathrm{pg} / \mathrm{mL}$ for Simoa, $15.6 \mathrm{pg} / \mathrm{mL}$ for the ECL assay, and $78.0 \mathrm{pg} / \mathrm{mL}$ for the ELISA. Correlations between paired CSF and serum samples were strongest for Simoa $(r=0.88, p<0.001)$ and

${ }^{a}$ Kaj Blennow and Henrik Zetterberg contributed equally to this work.

*Corresponding author: Jens Kuhle, MD, PhD, Neurology, Departments of Medicine, Biomedicine and Clinical Research, University Hospital Basel, Basel, Switzerland,

Phone: +41 (0)613287191; Fax: +41 (0)612654100,

E-mail: jens.kuhle@usb.ch

Christian Barro, Tobias Derfuss and Raija Lindberg: Neurology, Departments of Medicine, Biomedicine and Clinical Research, University Hospital Basel, Basel, Switzerland

Ulf Andreasson, Åsa Sandelius, Victor Liman and Kaj Blennow: Institute of Neuroscience and Physiology, Department of Psychiatry and Neurochemistry, The Sahlgrenska Academy, University of Gothenburg, Mölndal, Sweden

Niklas Norgren: UmanDiagnostics, Umeå, Sweden

Henrik Zetterberg: Institute of Neuroscience and Physiology, Department of Psychiatry and Neurochemistry, The Sahlgrenska Academy, University of Gothenburg, Mölndal, Sweden; and UCL Insitute of Neurology, Department of Psychiatry and Neurochemistry, Queen Square, London, UK the ECL assay $(r=0.78, p<0.001)$ and weaker for ELISA measurements $(r=0.38, p=0.030)$. CSF NfL measurements between the platforms were highly correlated $(r=1.0$, $\mathrm{p}<0.001)$. Serum NfL levels were highly related between ECL assay and Simoa $(r=0.86, p<0.001)$, and this was less visible between ELISA-ECL assay $(r=0.41, p=0.018)$ and ELISA-Simoa $(\mathrm{r}=0.43, \mathrm{p}=0.013)$. Multiple sclerosis (MS) patients had significantly higher serum NfL levels than controls when measured with Simoa $(p=0.001)$ but not with the other platforms.

Conclusions: We found Simoa to be more sensitive than ELISA or the ECL assay. Our results support the feasibility of quantifying NfL in serum; the results correlate with the more-established CSF NfL test. The highly sensitive Simoa technology deserves further studies in larger patient cohorts to clarify whether serum NfL could be used in the future to measure disease severity and determine prognosis or response to treatment interventions in neurological diseases.

Keywords: immunoassay; neurodegeneration; neurofilament light chain; serum.

\section{Introduction}

Highly sensitive methods for detecting soluble biomarkers for neuro-axonal damage are urgently needed in neurodegenerative diseases. Neurofilaments (Nf) are highly specific major structural proteins of neurons consisting predominantly of three Nf subunits: Nf light (NfL), Nf medium (NfM), and Nf heavy (NfH) chains [1]. Disruption to the axonal membrane releases $\mathrm{Nf}$ into the interstitial fluid and eventually into the cerebrospinal fluid (CSF) and blood. Therefore, blood Nf levels could potentially be useful for both predicting and monitoring disease progression and for assessing the efficacy and/or toxicity of future neuroprotective treatment strategies. Numerous previous studies have demonstrated the presence of $\mathrm{NfH}$ 
and NfL in CSF in a wide range of neurological diseases $[2,3]$; however, obtaining longitudinal CSF samples is relatively invasive, precluding the broader clinical use of Nf. In contrast, serial blood samples can be readily collected; hence, reliable quantification of NfL in blood would be a major stride towards a biomarker of the course of neurodegeneration.

A commercially available ELISA (UmanDiagnostics NF-light assay ELISA) uses two highly specific, non-competing monoclonal antibodies (mAB47:3 and mAB2:1) to quantify soluble NfL, but this assay is not recommended for blood measurements by the producer [4]. Electrochemiluminescence (ECL)-based assays are known to be highly sensitive, exhibit a broad dynamic range, and require small sample volume. We have recently developed an ECL immunoassay suitable for NfL measurements in serum based on these mABs (ECL assay) [5]. Single-molecule array (Simoa) technology for digital immunoassays has the potential to improve sensitivity significantly further $[6,7]$. The aim of this study was to compare the ELISA and ECL assay for NfL with a newly developed method based on Simoa technology using the same set of antibodies in clinically relevant and matched CSF and serum samples.

\section{Materials and methods}

\section{CSF and serum samples}

We selected pairs of CSF and serum samples based on previous serum NfL measurements with the ECL NfL assay: nine CSF and serum pairs with high (50-1800 pg/mL, high), eight with medium (5-15 pg/mL, medium), and seven with low ( $<4 \mathrm{pg} / \mathrm{mL}$, low) serum NfL levels. In the group of patients with high NfL levels, four patients suffered from amyotrophic lateral sclerosis (ALS), three had GuillainBarré syndrome (GBS), and one patient each had a major stroke or acute disseminated encephalomyelitis (ADEM). The group of patients with medium NfL levels consisted of three patients with multiple system atrophy (two Parkinsonian type and one cerebellar type), two patients each with non-infectious myelitis or stroke, and one patient with recurrent aseptic meningitis; the low NfL level group included four patients with tension-type headaches and one patient each with cryptogenic epilepsy, encephalopathy due to cannabis consumption and a radiologically isolated syndrome (RIS). In addition, we included nine CSF and serum pairs from patients with multiple sclerosis (MS) that had not been measured before. Six patients had a relapsing remitting disease course [median expanded disability status scale (EDSS): 6.5, interquartile range (IQR): 4.6-7.0, five patients with an acute relapse at sampling with onset of new symptoms within 30 days] and three were in the secondary progressive disease stage (median EDSS: 7.0, IQR: 6.5-7.5, three with acute relapse).

Samples were collected during routine diagnostic investigations as indicated by the treating physicians and collected and processed at room temperature within two hours (Neurology, University Hospital Basel, Switzerland). Serum samples were spun at $2000 \times \mathrm{g}$ and CSF samples at $400 \times g$ at room temperature for $10 \mathrm{~min}$, aliquoted in polypropylene tubes and stored at $-80^{\circ} \mathrm{C}[8]$.

\section{ELISA and ECL NfL assay}

The NF-light assay ELISA was performed as described by the manufacturer. The ECL assay [5] was slightly modified: Coating was done with $0.05 \mathrm{M}$ carbonate-bicarbonate buffer ( $\mathrm{pH}$ 9.6) at $4{ }^{\circ} \mathrm{C}$. Nonspecific binding sites were blocked with $100 \mu \mathrm{L}$ of TBS, containing 3\% milk powder, per well for $1 \mathrm{~h}$. We used $25 \mu \mathrm{L}$ of TBS containing $1 \%$ milk powder, $0.1 \%$ Tween-20, and 600/300 $\mu \mathrm{g} / \mathrm{mL}$ HeteroBlock ${ }^{\circledR}$ (Omega Biologicals, Bozeman, MT, USA) as sample diluent. Calibrators were prepared in TBS containing $1 \%$ milk powder, $0.1 \%$ Tween20, and $300 \mu \mathrm{g} / \mathrm{mL}$ HeteroBlock ${ }^{\circledR}$.

\section{Simoa NfL assay}

The Simoa NfL assay was established using the NF-light assay ELISA kit from UmanDiagnostics (UmanDiagnostics, Umeå, Sweden), transferred onto the Simoa platform with a homebrew kit (Quanterix Corp, Boston, MA, USA), and detailed instructions can be found in the Simoa Homebrew Assay Development Guide (Quanterix). In short, paramagnetic carboxylated beads (Quanterix) were activated using 1-ethyl-3-(3-dimethylaminopropyl) carbodiimide (EDAC) (Quanterix) by adding 5\% (v/v) $10 \mathrm{mg} / \mathrm{mL}$ EDAC to a magnetic beads solution with $1.4 \times 10^{6}$ beads/ $\mu \mathrm{L}$. Following a $30-\mathrm{min}$ incubation at room temperature (RT), the beads were washed using a magnetic separator, and an initial volume, i.e. EDAC+beads solution volumes in the previous step, of $0.3 \mathrm{mg} / \mathrm{mL}$ ice cold solution of the monoclonal capture antibody (mAB47:3, UmanDiagnostics) was added. After a 2-h incubation on a mixer (2000 rpm, Multi-Tube Vortexer, Allsheng, China) at RT, the beads were washed and an initial reaction volume of blocking solution was added. After three washes, the conjugated beads were suspended and stored at $4{ }^{\circ} \mathrm{C}$ pending analysis. The monoclonal detection antibody $(1 \mathrm{mg} / \mathrm{mL}$, mAB2:1, UmanDiagnostics) was biotinylated by adding 3\% (v/v) 3.4 mM EZ-Link ${ }^{\mathrm{TM}}$ NHS-PEG4-Biotin (Quanterix) followed by a 30-min incubation at RT. Free biotin was removed using spin filtration (Amicon ${ }^{\circledR}$ Ultra-2, $50 \mathrm{kDa}$, Sigma) and the biotinylated antibody was stored at $4{ }^{\circ} \mathrm{C}$ pending analysis. The assay was run on a Simoa HD-1 instrument (Quanterix) using a 2-step Assay Dilution 2.0 protocol using $25 \mu \mathrm{L}$ conjugated beads, $75 \mu \mathrm{L}$ diluent [PBS; 0.1\% Tween-20; 2\% BSA; 10 mcg/mL TRU Block (Meridian Life Science, Inc., Memphis, TN, USA)], $20 \mu \mathrm{L}$ biotinylated antibody, and $25 \mu \mathrm{L}$ sample (or calibrator), which was followed by a 47 cadances incubation ( 1 cadance $=45 \mathrm{~s}$ ). After washing, $100 \mu \mathrm{L}$ of streptavidinconjugated $\beta$-galactosidase (Quanterix) was added, followed by a 7-cadence incubation and a wash. Prior to reading, $25 \mu \mathrm{L}$ Resorufin $\beta$-D-galactopyranoside (Quanterix) was added. The calibrator curve was constructed using the standard from the NF-light assay ELISA (NF-light ${ }^{\circledR}$, UmanDiagnostics).

All measurements (ELISA, ECL assay, Simoa) were performed by board-certified laboratory technicians, who were masked to clinical data using one batch of reagents. 


\section{Ethics statement}

Written informed consent was obtained from all patients, and the study was approved by the Common Institutional Review Board of the Cantons of Basel.

\section{Analytical and statistical analysis}

Samples below the lowest calibrator reaching acceptance criteria [calibrator: accuracy: $80 \%-120 \%$, coefficients of variation (CVs) of duplicate determination $\leq 20 \%$ ] were assigned the concentration of the lowest calibrator reaching these criteria [9]. We used the concentration of the lowest calibrator fulfilling these acceptance criteria as an estimate of the "analytical sensitivity" of each platform rather than the "limit of detection" that is often incorrectly used to describe assay "sensitivity". The lower limit of quantification was not formally assessed for each of the platforms in this comparison study [10]. Samples above the highest calibrator $(10,000 \mathrm{pg} / \mathrm{mL})$ were assigned a concentration of $10,000 \mathrm{pg} / \mathrm{mL}$. Repeatability (within-run precision) and intermediate precision (between-run precision) were calculated according to [11]. Intra-assay CVs were assessed between the concentrations of duplicates of the measured samples. All measurements on the Meso Scale Diagnostics (MSD) and Simoa platforms were done in duplicates; the ELISA measurements were performed in single measurements, since based on large previous experience, intermediate precision and repeatability for the ELISA are below $20 \%$ and $10 \%$, respectively.

Data are described by median and IQR if not stated otherwise. NfL levels were compared between MS and each of the other groups (low, medium, high) by the Mann-Whitney test. Spearman correlation coefficients were calculated between NfL levels in CSF and serum and between the different platforms. All analyses were performed using GraphPad Prism 6.00 for Windows [GraphPad software, La Jolla, CA, USA (www.graphpad.com)] and IBM SPSS Statistics, version 22.

\section{Results}

\section{Sensitivity of ELISA, ECL assay, and Simoa}

Analytical sensitivity was $0.62 \mathrm{pg} / \mathrm{mL}$ for Simoa, $15.6 \mathrm{pg} /$ $\mathrm{mL}$ for the ECL assay, and $78.0 \mathrm{pg} / \mathrm{mL}$ for the ELISA. All CSF samples in all assays had concentrations above these levels. Eighteen of $33(54.5 \%)$ serum measurements and 20 of $33(60.6 \%)$ were below sensitivity in the ELISA and ECL assay, respectively (Table 1). Of note, several ELISA serum measurements showed very high NfL concentrations also in low and medium (low: $1 / 7$ with very high NfL concentration, measured concentration: $3201 \mathrm{pg} / \mathrm{mL}$; medium: 4/8, measured concentrations: $199,219,517$, and $1209 \mathrm{pg} / \mathrm{mL}$ ). These five samples were all below analytical sensitivity when measured by the ECL assay and had low concentration with Simoa (range 7-18 pg/mL).

\section{Intermediate precision, repeatability, and sample CVs for the ECL assay and Simoa}

All samples were measured on the same day. Based on results from the previous 15 runs performed on separate days, intermediate precision/repeatability were $6.6 \% / 3.6 \%$ (sample with mean concentration $72.8 \mathrm{pg} / \mathrm{mL}$ ), 8.8\%/6.4\% (52.3 pg/mL) and 14.8\%/9.2\% (9.1 pg/mL), respectively, for the ECL assay. All sample CVs of duplicate measurements were below $10.8 \%$.

Since the protocol for the Simoa assay is still under development, mainly due to planned upgrades of the platform, no complete formal validation has been performed. Based on results from the previous 13 runs using the current protocol, intermediate precision/repeatability was $17.0 \% / 6.6 \%$, respectively (sample with mean concentration of $64 \mathrm{pg} / \mathrm{mL}$ ). All sample CVs of duplicate measurements were below $12.5 \%$.

\section{Correlations between CSF and serum NfL}

Correlations between paired CSF and serum samples were strongest for Simoa $(r=0.88, p<0.001$, Figure $1 C)$ and the ECL assay $(r=0.78, p<0.001$, Figure $1 B)$. This was less clear for the ELISA measurements $(r=0.38, p=0.030$, Figure $1 \mathrm{~A})$ (Table 1).

\section{Correlations between ELISA, MSD and Simoa measurements}

\section{CSF}

CSF NfL measurements on the three different platforms were highly correlated (ELISA-ECL assay: $r=1.0, p<0.001$, Figure 1D; ELISA-Simoa: $\mathrm{r}=1.0, \mathrm{p}<0.001$, Figure 1E; and ECL assay-Simoa: $r=1.0, p<0.001$, Figure $1 F$ ).

\section{Serum}

For serum measurements, NfL levels were highly correlated between the ECL assay and Simoa $(r=0.86, p<0.001$, Figure 1I), whereas this relation was less strong for ELISAECL assay $(r=0.41, p=0.018$, Figure $1 \mathrm{G})$ and ELISA-Simoa $(r=0.43, p=0.013$, Figure $1 \mathrm{H})$. 
Table 1: Results of CSF and serum NfL measurements in ELISA, ECL assay and Simoa.

\begin{tabular}{|c|c|c|c|}
\hline & ELISA & ECL assay & Simoa \\
\hline \multicolumn{4}{|l|}{$\operatorname{CSF}(n=33)$} \\
\hline Sensitivity & $78.0 \mathrm{pg} / \mathrm{mL}$ & $15.6 \mathrm{pg} / \mathrm{mL}$ & $0.62 \mathrm{pg} / \mathrm{mL}$ \\
\hline $\mathrm{NfL}, \mathrm{pg} / \mathrm{mL}$ & $1074(426.0-3051.5)$ & $965(345-2727)$ & $1649(558.5-4997.5)$ \\
\hline $\mathrm{N}$ below sensitivity & 0 & 0 & 0 \\
\hline Low $(n=7)$ & 317.0 & 272.0 & 475.0 \\
\hline Medium $(\mathrm{n}=8)$ & 715.5 & 631.5 & 1009.5 \\
\hline High $(n=9)$ & 5611.0 & 5333.0 & 7854.0 \\
\hline MS $(n=9)$ & 2112.0 & 1834.0 & 3600.0 \\
\hline Pair-wise & Low: $p=0.005$ & Low: $p=0.002$ & Low: $p=0.005$ \\
\hline \multirow[t]{2}{*}{ comparison with MS } & Med.: $p=0.074$ & Med.: $p=0.046$ & Med.: $p=0.059$ \\
\hline & High: $p=0.063$ & High: $p=0.050$ & High: $p=0.063$ \\
\hline \multicolumn{4}{|l|}{ Serum $(n=33)$} \\
\hline $\mathrm{NfL}, \mathrm{pg} / \mathrm{mL}$ & $78.0(78.0-252.0)$ & $15.6(15.6-62.5)$ & $22.0(12.5-54.5)$ \\
\hline $\mathrm{N}$ below sensitivity & $18(54.5)$ & $20(60.6)$ & $0(0)$ \\
\hline Low & $78.0^{\mathrm{a}}$ & $15.6^{\mathrm{b}}$ & 8.0 \\
\hline Medium & $138.0^{\mathrm{a}}$ & $15.6^{b}$ & 16.5 \\
\hline High & 233.0 & 88.0 & 103.0 \\
\hline MS & $78.0^{\mathrm{a}}$ & $15.6^{\mathrm{b}}$ & 25.0 \\
\hline Pair-wise & Low: $p=0.837$ & Low: $p=0.142$ & Low: $p=0.001$ \\
\hline \multirow[t]{2}{*}{ comparison with MS } & Med.: $p=0.606$ & Med.: $p=0.074$ & Med.: $p=0.074$ \\
\hline & High: $p=0.040$ & High: $p<0.001$ & High: $p=0.003$ \\
\hline \multicolumn{4}{|l|}{ CSF/serum correlations } \\
\hline Spearman R and p & $0.38,0.030$ & $0.78,<0.001$ & $0.88,<0.001$ \\
\hline \multicolumn{4}{|c|}{ CSF: Spearman R and $p$ for correlation between ELISA, ECL assay and Simoa } \\
\hline ELISA & - & & \\
\hline ECL assay & $1.0,<0.001$ & - & \\
\hline Simoa & $1.0,<0.001$ & $1.0,<0.001$ & - \\
\hline \multicolumn{4}{|c|}{ Serum: Spearman R and p for correlation between ELISA, ECL assay and Simoa } \\
\hline ELISA & - & & \\
\hline ECL assay & $0.41,0.018$ & - & \\
\hline Simoa & $0.43,0.013$ & $0.86,<0.001$ & - \\
\hline
\end{tabular}

aBelow lowest calibrator $(78.0 \mathrm{pg} / \mathrm{mL})$ : low: $6 / 7$ samples (1/7 measured concentration $3201 \mathrm{pg} / \mathrm{mL})$; medium: 4/8 (4/8: $199 \mathrm{pg} / \mathrm{mL}$, $219 \mathrm{pg} / \mathrm{mL}, 517 \mathrm{pg} / \mathrm{mL}$, and $1209 \mathrm{pg} / \mathrm{mL}) ; \mathrm{MS}: 7 / 9$ (2/9: $5595 \mathrm{pg} / \mathrm{mL}$ and $1891 \mathrm{pg} / \mathrm{mL}) .{ }^{b}$ Below lowest calibrator (15.6 pg/mL): low: 7/7; medium: 8/8; MS: 5/9. CSF, cerebrospinal fluid; ECL, electrochemiluminescence; MS, multiple sclerosis; NfL, neurofilament light chain.

\section{Comparison between MS and low, medium, Discussion and high}

\section{CSF}

MS patients had CSF NfL levels above low in all three platforms (ELISA: $\mathrm{p}=0.005$, ECL assay: $\mathrm{p}=0.002$, Simoa: $\mathrm{p}=0.005)$. The difference between MS and medium or high only reached significance for the ECL assay ( $p=0.046$ and $\mathrm{p}=0.050$, respectively, Table 1 ).

\section{Serum}

Interestingly, the increase in serum NfL in MS vs. low only reached significance in Simoa ( $p=0.001$, Table 1). MS had significantly lower serum NfL than high in all three assays (ELISA: $\mathrm{p}=0.04$, ECL assay: $\mathrm{p}<0.001$, Simoa: $\mathrm{p}=0.003$ ).
We found the Simoa platform to be 126- and 25-fold more sensitive than ELISA and the ECL assay, respectively, to quantify NfL. CSF and serum NfL measurements were highly correlated in the ECL assay and Simoa measurements, whereas this relationship was weaker with ELISA. The correlation coefficients for CSF NfL measurements between the different platforms were 1.0. Conversely, only the serum NfL results from the ECL assay and Simoa showed a similar high relationship.

The Simoa technology relies on single-molecule arrays and the simultaneous counting of singulated capture microbeads $[6,7]$. In contrast to analog immunoassays where the enzyme-substrate reaction is conducted in relatively large reaction volumes (50$100 \mu \mathrm{L}$ ), Simoa restricts the diffusion of the fluorescent 
A
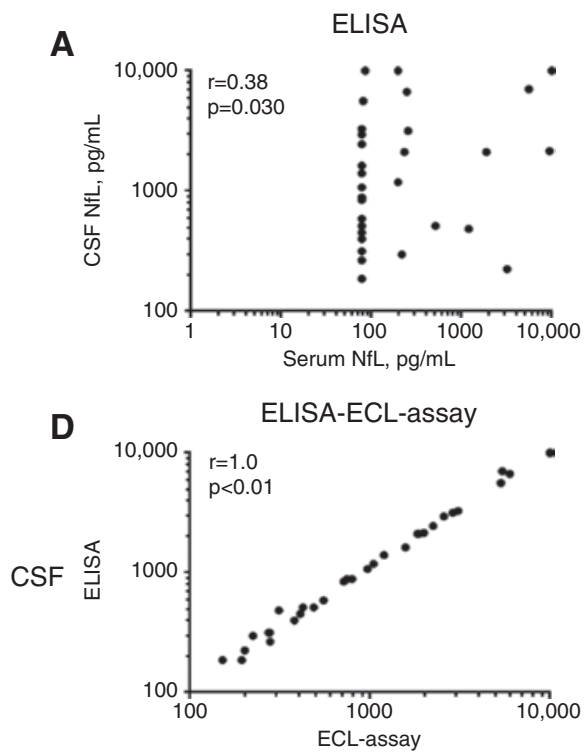

G

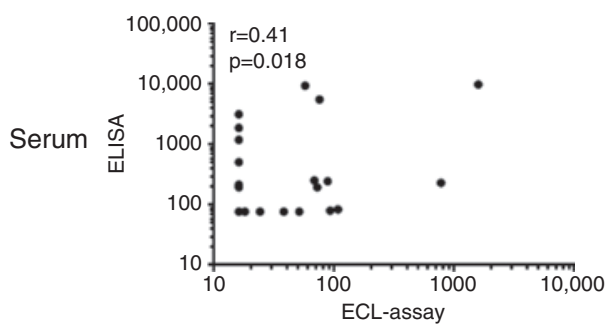

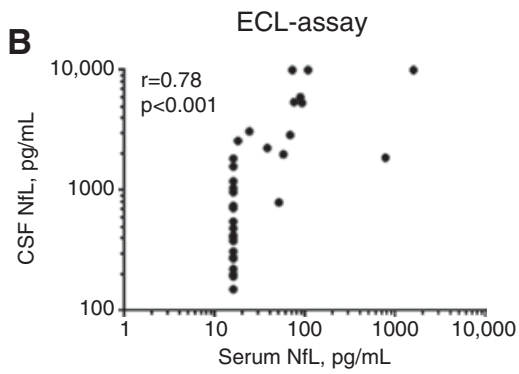
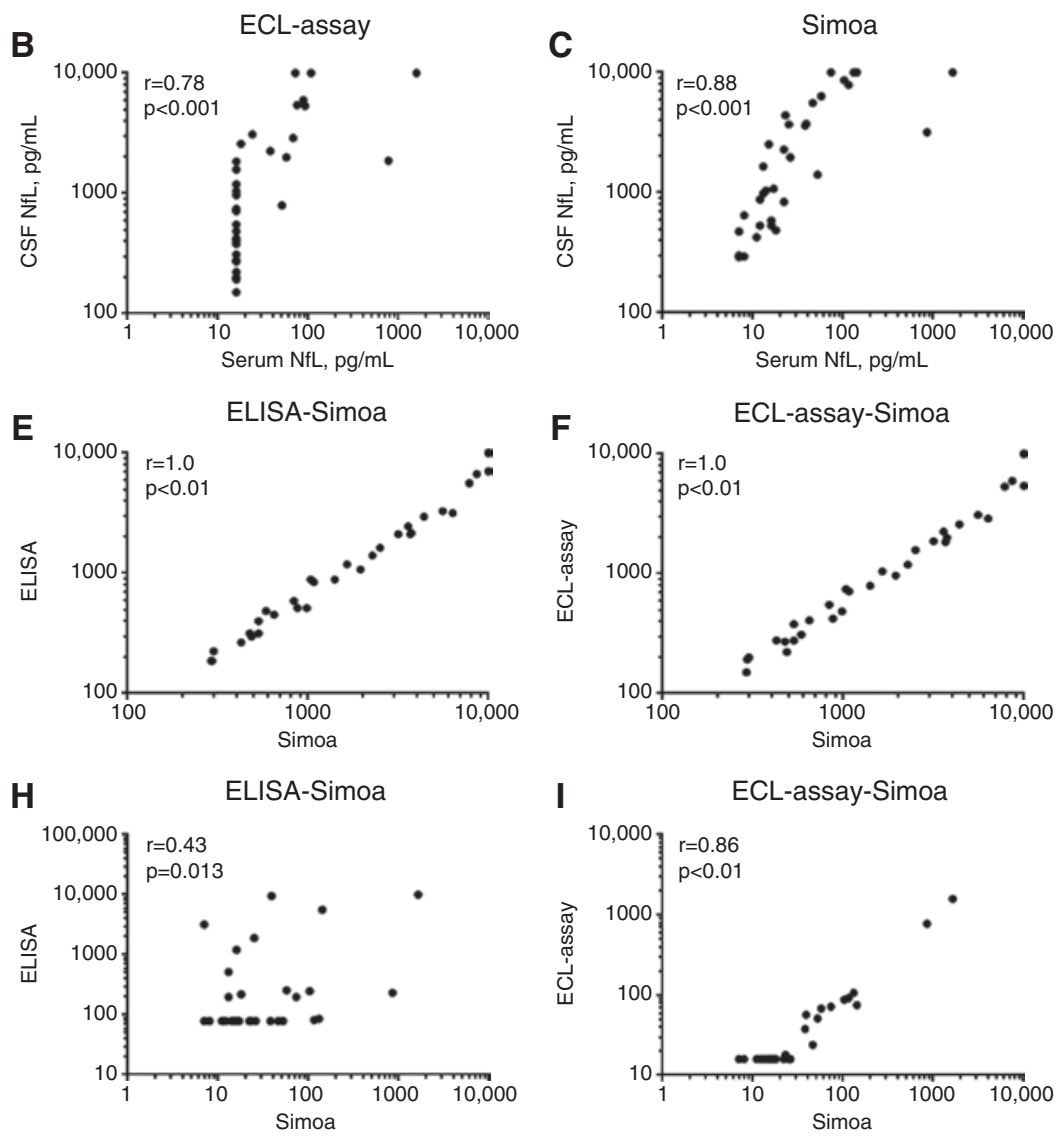

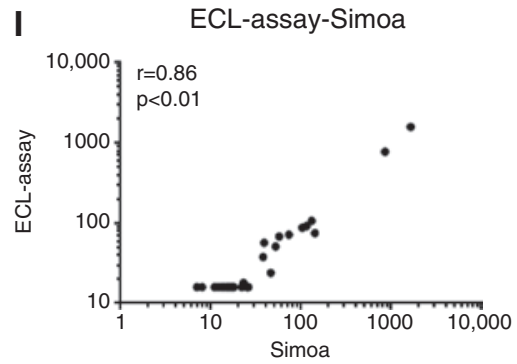

Figure 1: CSF and serum NfL correlation in different assays (A-C), associations between $\mathrm{NfL}$ measurements on different platforms in CSF (D-F) and serum (G-I).

Correlations between paired CSF and serum samples were strongest for Simoa ( $r=0.88, p<0.001$, Figure 1C), and the ECL assay ( $r=0.78$, $p<0.001$, Figure 1B). This was less clear for the ELISA measurements $(r=0.38, p=0.030$, Figure 1A). CSF NfL measurements on the three different platforms were highly correlated: ELISA-ECL assay: $r=1.0, p<0.001$, Figure 1D; ELISA-Simoa: $r=1.0, p<0.001$, Figure 1E; and ECL assay-Simoa: $r=1.0, p<0.001$, Figure 1F. For serum measurements, NfL levels were highly correlated between $E C L$ assay and Simoa ( $r=0.86$, $p<0.001$, Figure 1), whereas this relation was weaker for ELISA-ECL assay $(r=0.41, p=0.018$, Figure $1 G)$ and ELISA-Simoa $(r=0.43, p=0.013$, Figure $1 \mathrm{H})$.

molecules by femtoliter-sized wells that can be counted with a camera simultaneously in thousands of microwells. The counting of active and inactive wells constitutes a digital signal corresponding to the presence or absence of single enzyme molecules. This gain in sensitivity permits the use of low quantities of the labeling reagent, which lowers nonspecific interactions and increases signal-background ratios [7]. Indeed, we found the Simoa platform to be more than 100-fold more sensitive $(0.62 \mathrm{pg} / \mathrm{mL})$ than ELISA $(78.0 \mathrm{pg} / \mathrm{mL})$, and importantly, also 25 -fold more sensitive than our previously described and validated ECL assay $(15.6 \mathrm{pg} / \mathrm{mL})$ [5]. For any potential future clinical application, it is of utmost importance to note that all serum samples were well in the measurable range of the Simoa NfL assay, with the lowest serum sample displaying a concentration of $2.0 \mathrm{pg} / \mathrm{mL}, 3.2$-fold above the sensitivity of the assay. In contrast, more than $50 \%$ of the samples were not reliably quantifiable by the ECL assay and ELISA in our study.

Brain proteins access the blood flow either via CSF drainage in the venous blood or by diffusion through the blood-brain barrier. We have previously shown associations between CSF and serum in ALS, Alzheimer's disease, GBS and healthy controls [5, 12], which is in line with the present results. Indeed, CSF and serum measurements were highly correlated with the ECL assay $(r=0.78, p<0.001$, Figure $1 B)$ and Simoa $(r=0.88, p<0.001$, Figure $1 \mathrm{C}$ ), but less so with ELISA ( $\mathrm{r}=0.38, \mathrm{p}=0.030$, Figure 1A). We postulate that lower sensitivity together with unexpected high serum NfL measurements in some of the serum samples, caused by matrix effects like the presence of heterophilic antibodies, contributed to this smaller correlation between CSF and serum ELISA measurements 
(see footnote Table 1) [13]. Heterophilic antibodies are also likely the cause for the apparently higher rate of measurements below sensitivity with the ECL assay compared with the ELISA, which does not contain a blocker for this type of interference.

NfL measurements in CSF were perfectly correlated between the three platforms (Figure 1D-F). Of note, absolute CSF NfL levels tended to be higher with Simoa $(1649 \mathrm{pg} / \mathrm{mL})$, whereas concentrations measured by ELISA $(1074 \mathrm{pg} / \mathrm{mL})$ and the ECL assay $(965 \mathrm{pg} / \mathrm{mL})$ were more similar. This is an important observation and points to the need for standardization of calibrators and further work to allow direct comparability of concentrations between different platforms, centres, and studies. Also, despite the fact that our results seem to be, to some extent, expected (as all three platforms used the same mABs), several examples from the peerreviewed literature clearly show the difficulty of reproducing findings even with identical reagents and platforms [14, 15]. These examples clearly point to the importance of thorough biomarker validation, including longitudinal cohort studies, before implementing biomarkers for research, clinical trials, or routine use in clinical practice [16].

Correlations between ELISA serum measurements and ECL assay or Simoa were visible and significant, but limited likely by the ELISA's shortcomings described above (Figure 1G-H). Serum measurements between ECL assay and Simoa were highly associated (Figure 1I), with the ECL assay's limitation that it cannot quantify concentrations below $15.6 \mathrm{pg} / \mathrm{mL}$ (20 of 33 samples). Our data confirm that serum NfL can be reproducibly measured on different sufficiently sensitive assay platforms and support the use of NfL measurements as a biomarker of neuronal damage also in the blood compartment.

We attempted to investigate CSF and serum NfL levels in MS vs. the preselected defined range samples (low, medium, high). We saw significantly higher CSF NfL levels in MS vs. low for all three platforms. Conversely, serum NfL in MS was only significantly higher than low for the Simoa measurements, whereas neither ELISA nor the ECL assay allowed quantification of any samples from the low or medium category. With the ECL assay, it was possible to quantify five of nine MS samples vs. zero with the ELISA (Table 1).

In conclusion, our results support the feasibility of quantifying NfL in CSF and serum samples as a measure of axonal injury. The highly sensitive Simoa technology especially deserves further studies in large and wellcharacterized longitudinal patient cohorts. Large studies including longitudinal sampling will also be needed to clarify whether serum NfL can be used in the future to measure disease severity and determine prognosis or/and response to treatment interventions in various neurological diseases.

Acknowledgments: We thank M. Limberg for technical assistance.

Author contributions: All the authors have accepted responsibility for the entire content of this submitted manuscript and approved submission.

Research funding: The study was supported by grants from the Swiss MS Society, Swiss National Science Foundation (Grant/Award Number: '320030_160221'), Bayer (Switzerland) AG, Genzyme, Novartis, Swedish Research Council, VINNOVA, the Torsten Söderberg Foundation, the Knut and Alice Wallenberg Foundation, Swedish State Support for Clinical Research, and Frimurarestiftelsen.

Employment or leadership: None declared.

Honorarium: None declared.

Competing interests: The funding organization(s) played no role in the study design; in the collection, analysis, and interpretation of data; in the writing of the report; or in the decision to submit the report for publication.

\section{References}

1. Teunissen CE, Khalil M. Neurofilaments as biomarkers in multiple sclerosis. Mult Scler 2012;18:552-6.

2. Deisenhammer F, Egg R, Giovannoni G, Hemmer B, Petzold A, Sellebjerg F, et al. EFNS guidelines on disease-specific CSF investigations. Eur J Neurol 2009;16:760-70.

3. Teunissen CE, Iacobaeus E, Khademi M, Brundin L, Norgren N, Koel-Simmelink MJ, et al. Combination of CSF N-acetylaspartate and neurofilaments in multiple sclerosis. Neurology 2009;72:1322-9.

4. Norgren N, Karlsson JE, Rosengren L, Stigbrand T. Monoclonal antibodies selective for low molecular weight neurofilaments. Hybrid Hybridomics 2002;21:53-9.

5. Gaiottino J, Norgren N, Dobson R, Topping J, Nissim A, Malaspina $A$, et al. Increased neurofilament light chain blood levels in neurodegenerative neurological diseases. PLoS One 2013;8:e75091.

6. Rissin DM, Kan CW, Campbell TG, Howes SC, Fournier DR, Song L, et al. Single-molecule enzyme-linked immunosorbent assay detects serum proteins at subfemtomolar concentrations. Nat Biotechnol 2010;28:595-9.

7. Wilson DH, Rissin DM, Kan CW, Fournier DR, Piech T, Campbell TG, et al. The simoa HD-1 analyzer: a novel fully automated digital immunoassay analyzer with single-molecule sensitivity and multiplexing. J Lab Autom 2015 [Epub ahead of print].

8. Teunissen CE, Petzold A, Bennett JL, Berven FS, Brundin L, Comabella $\mathrm{M}$, et al. A consensus protocol for the standardization of cerebrospinal fluid collection and biobanking. Neurology 2009;73:1914-22.

9. Valentin MA, Ma S, Zhao A, Legay F, Avrameas A. Validation of immunoassay for protein biomarkers: bioanalytical study plan 
implementation to support pre-clinical and clinical studies. J Pharm Biomed Anal 2011;55:869-77.

10. Lee JW, Devanarayan V, Barrett YC, Weiner R, Allinson J, Fountain $\mathrm{S}$, et al. Fit-for-purpose method development and validation for successful biomarker measurement. Pharm Res 2006;23:312-28.

11. Andreasson U, Perret-Liaudet A, van Waalwijk van Doorn LJ, Blennow K, Chiasserini D, Engelborghs S, et al. A Practical Guide to Immunoassay Method Validation. Front Neurol 2015;6:179.

12. Lu CH, Macdonald-Wallis C, Gray E, Pearce N, Petzold A, Norgren $\mathrm{N}$, et al. Neurofilament light chain: a prognostic biomarker in amyotrophic lateral sclerosis. Neurology 2015;84:2247-57.

13. Lundberg M, Curbo S, Reiser K, Masterman T, BraeschAndersen S, Arestrom I, et al. Methodological aspects of ELISA analysis of thioredoxin 1 in human plasma and cerebrospinal fluid. PLoS One 2014;9:e103554.

14. Kuhle J, Pohl C, Mehling M, Edan G, Freedman MS, Hartung $\mathrm{HP}$, et al. Lack of association between antimyelin antibodies and progression to multiple sclerosis. N Engl I Med 2007;356:371-8.

15. Brickshawana A, Hinson SR, Romero MF, Lucchinetti C, Guo Y, Buttmann M, et al. Investigation of the KIR4.1 potassium channel as a putative antigen in patients with multiple sclerosis: a comparative study. Lancet Neurol 2014;13:795-806.

16. Teunissen CE, Malekzadeh A, Leurs C, Bridel C, Killestein J. Body fluid biomarkers for multiple sclerosis-the long road to clinical application. Nat Rev Neurol 2015;11:585-96. 